\title{
Linear Relations and their Breakdown in High Energy Massive String Scatterings in Compact Spaces
}

\author{
Jen-Chi Lee* \\ Institute of Physics, University of Tokyo, \\ Komaba, Meguro-ku, Tokyo 153-8902, Japan and \\ Department of Electrophysics, National Chiao-Tung University, Hsinchu, Taiwan, R.O.C. \\ Yi Yangt \\ Institute of Physics, University of Tokyo, \\ Komaba, Meguro-ku, Tokyo 153-8902, Japan and \\ Physics Division, National Center for Theoretical Sciences, Hsinchu, Taiwan, R.O.C.
}

(Dated: December 7, 2018)

\begin{abstract}
We calculate high energy massive scattering amplitudes of closed bosonic string compactified on the torus. For each fixed mass level with given quantized and winding momenta $\left(\frac{m}{R}, \frac{1}{2} n R\right)$, we obtain infinite linear relations among high energy scattering amplitudes of different string states. For some kinematic regimes, we discover that linear relations with $N_{R}=N_{L}$ break down and, simultaneously, the amplitudes enhance to power-law behavior instead of the usual expoential falloff behavior at high energies. It is the space-time T-duality symmetry that plays a role here. This result is consistent with the coexistence of the linear relations and the softer exponential fall-off behavior of high energy string scattering amplitudes as we pointed out prevously. It is also reminiscent of our previous work on the power-law behavior of high energy string/domain-wall scatterings.
\end{abstract}

*Email: jcclee@cc.nctu.edu.tw; (on leave of absence from NCTU)

$\dagger$ Email: yyang@phys.cts.nthu.edu.tw; (on leave of absence from NCTS) 


\section{Contents}

I. Introduction

\section{String compactified on torus}

A. Winding string and kinematic setup

B. Four-tachyon scatterings with $N_{R}=N_{L}=0$

\section{High energy behaviors}

A. High energy massive scatterings for general $N_{R}+N_{L}$

B. The infinite linear relations in the GR

C. Power-law and breakdown of the infinite linear relations in the MR

\section{Acknowledgments}

\section{References}

\section{INTRODUCTION}

It is well known that there are two fundamental characteristics of high energy string scattering amplitudes, which make them very different from field theory scatterings. These are the softer exponential fall-off behavior (in contrast to the power-law behavior of field theory scatterings) and the existence of infinite Regge-pole structure in the form factor of the high energy string scattering amplitudes.

Recently high-energy, fixed angle behavior of string scattering amplitudes \1, 2, 3n was intensively reinvestigated for massive string states at arbitrary mass levels [4, 5, 6, 7, 8, 9, 10, 11, 12]. An infinite number of linear relations, or stringy symmetries, among string scattering amplitudes of different string states were obtained. An important new ingredient of these calculations is the zero-norm states (ZNS) [13, 14, 15] in the old covariant first quantized (OCFQ) string spectrum. The existence of these infinite linear relations constitutes the third fundamental characteristics of high energy string scatterings, which is not shared by the usual point-particle field theory scatterings. 
These linear relations persist for string scattered from generic D $p$-brane [16] except Dinstanton and Domain-wall. For the scattering of D-instanton, the form factor exhibits the well-known power-law behavior without Regge-pole structure, and thus resembles a field theory amplitude. For the special case of Domain-wall (D24-brane) scattering, it was discovered [17] recently that, in contrast to the common wisdom of exponential fall off behavior [18, 19], its form factor behaves as power-law with Regge-pole structure. This discovery makes Domain-wall scatterings a hybrid of string and field theory scatterings. Moreover, it was shown [17] that the linear relations break down for the Domain-wall scattering due to this unusual power-law behavior. This result gives a strong evidence that the existence of the infinite linear relations, or stringy symmetries, of high-energy string scattering amplitudes is responsible for the softer (exponential fall-off) high-energy string scatterings than the (power-law) field theory scatterings.

To further convince ourselves with the coexistence of the infinite linear relations and the softer exponential fall-off behavior of string scatterings at high energies, it is important to find more examples of high energy string scatterings, which show the unusual power-law behavior and, simultaneously, give the breakdown of the infinite linear relations. With this in mind, in this paper we calculate high energy massive scattering amplitudes of closed bosonic string with some coordinates compactified on the torus [20, 21]. For each fixed mass level with given quantized and winding momenta $\left(\frac{m}{R}, \frac{1}{2} n R\right)$, we obtain infinite linear relations among high energy scattering amplitudes of different string states. This result is reminiscent of the existence of an infinite number of massive soliton ZNS in the compactified string constructed in [22]. We then discover that, for some kinematic regime, so called Mende regime (MR), infinite linear relations with $N_{R}=N_{L}$ break down and, simultaneously, the amplitudes enhance to power-law behavior instead of the usual exponential fall-off behavior at high energies. It is the space-time T-duality symmetry that plays a role here.

The power-law behavior of high energy string scatterings in a compact space was first suggested by Mende [20]. Here we give an explicit calculation of the conjecture. Moreover, in addition to the high energy string/domain-wall scatterings mentioned above [17], our result in this paper gives another evidence to support the coexistence of the infinite linear relations and the softer exponential fall-off behavior of high energy string scattering amplitudes as we pointed out previously [17, 23]. The result also suggests that the infinite linear relations are closely related to the full $26 \mathrm{D}$ space-time symmetry of closed bosonic string theory. This 
paper is organized as following. In section II, we set up the kinematic for the compactified string and calculate the four-tachyon $\left(N_{R}=N_{L}=0\right)$ scattering amplitudes with arbitrary winding. In section III, we derive the infinite linear relations among high energy scattering amplitudes of different string states with given $\left(\frac{m}{R}, \frac{1}{2} n R\right)$ for each fixed mass level. We then discuss the power-law behavior of the amplitudes and breakdown of the infinite linear relations in the Mende regime. A brief conclusion is given in section IV.

\section{STRING COMPACTIFIED ON TORUS}

\section{A. Winding string and kinematic setup}

We consider 26D closed bosonic string with one coordinate compactified on $S^{1}$ with radius $R$. As we will see later, it is straightforward to generalize our calculation to more compactified coordinates. The closed string boundary condition for the compactified coordinate is (we use the notation in [24])

$$
X^{25}(\sigma+2 \pi, \tau)=X^{25}(\sigma, \tau)+2 \pi R n
$$

where $n$ is the winding number. The momentum in the $X^{25}$ direction is then quantized to be

$$
K=\frac{m}{R}
$$

where $m$ is an integer. The mode expansion of the compactified coordinate is

$$
X^{25}(\sigma, \tau)=X_{R}^{25}(\sigma-\tau)+X_{L}^{25}(\sigma+\tau),
$$

where

$$
\begin{aligned}
& X_{R}^{25}(\sigma-\tau)=\frac{1}{2} x+K_{R}(\sigma-\tau)+i \sum_{k=0} \frac{1}{k} \alpha_{k}^{25} e^{-2 i k(\sigma-\tau)}, \\
& X_{L}^{25}(\sigma+\tau)=\frac{1}{2} x+K_{L}(\sigma+\tau)+i \sum_{k=0} \frac{1}{k} \tilde{\alpha}_{k}^{25} e^{-2 i k(\sigma+\tau)} .
\end{aligned}
$$

The left and right momenta are defined to be

$$
K_{L, R}=K \pm L=\frac{m}{R} \pm \frac{1}{2} n R \Rightarrow K=\frac{1}{2}\left(K_{L}+K_{R}\right)
$$


and the mass spectrum can be calculated to be

$$
\left\{\begin{array}{c}
M^{2}=\left(\frac{m^{2}}{R^{2}}+\frac{1}{4} n^{2} R^{2}\right)+N_{R}+N_{L}-2 \equiv K_{L}^{2}+M_{L}^{2} \equiv K_{R}^{2}+M_{R}^{2} \\
N_{R}-N_{L}=m n
\end{array}\right.
$$

where $N_{R}$ and $N_{L}$ are the number operators for the right and left movers, which include the counting of the compactified coordinate. We have also introduced the left and the right level masses as

$$
M_{L, R}^{2} \equiv 2\left(N_{L, R}-1\right)
$$

Note that for the compactified closed string $N_{R}$ and $N_{L}$ are correlated through the winding modes.

In the center of momentum frame, the kinematic can be set up to be

$$
\begin{aligned}
& k_{1 L, R}=\left(+\sqrt{p^{2}+M_{1}^{2}},-p, 0,-K_{1 L, R}\right), \\
& k_{2 L, R}=\left(+\sqrt{p^{2}+M_{2}^{2}},+p, 0,+K_{2 L, R}\right), \\
& k_{3 L, R}=\left(-\sqrt{q^{2}+M_{3}^{2}},-q \cos \phi,-q \sin \phi,-K_{3 L, R}\right), \\
& k_{4 L, R}=\left(-\sqrt{q^{2}+M_{4}^{2}},+q \cos \phi,+q \sin \phi,+K_{4 L, R}\right)
\end{aligned}
$$

where $p \equiv|\tilde{\mathrm{p}}|$ and $q \equiv|\tilde{\mathrm{q}}|$ and

$$
\begin{aligned}
k_{i} & \equiv \frac{1}{2}\left(k_{i R}+k_{i L}\right), \\
k_{i}^{2} & =K_{i}^{2}-M_{i}^{2}, \\
k_{i L, R}^{2} & =K_{i L, R}^{2}-M_{i}^{2} \equiv-M_{i L, R}^{2} .
\end{aligned}
$$

With this setup, the center of mass energy $E$ is

$$
E=\frac{1}{2}\left(\sqrt{p^{2}+M_{1}^{2}}+\sqrt{p^{2}+M_{2}^{2}}\right)=\frac{1}{2}\left(\sqrt{q^{2}+M_{3}^{2}}+\sqrt{q^{2}+M_{4}^{2}}\right) .
$$

The conservation of momentum on the compactified direction gives

$$
m_{1}-m_{2}+m_{3}-m_{4}=0
$$

and T-duality symmetry implies conservation of winding number

$$
n_{1}-n_{2}+n_{3}-n_{4}=0 .
$$


One can easily calculate the following kinematic relations

$$
\begin{aligned}
-k_{1 L, R} \cdot k_{2 L, R} & =\sqrt{p^{2}+M_{1}^{2}} \cdot \sqrt{p^{2}+M_{2}^{2}}+p^{2}+\vec{K}_{1 L, R} \cdot \vec{K}_{2 L, R} \\
& =\frac{1}{2}\left(s_{L, R}+k_{1 L, R}^{2}+k_{2 L, R}^{2}\right)=\frac{1}{2} s_{L, R}-\frac{1}{2}\left(M_{1 L, R}^{2}+M_{2 L, R}^{2}\right), \\
-k_{2 L, R} \cdot k_{3 L, R} & =-\sqrt{p^{2}+M_{2}^{2}} \cdot \sqrt{q^{2}+M_{3}^{2}}+p q \cos \phi+\vec{K}_{2 L, R} \cdot \vec{K}_{3 L, R} \\
& =\frac{1}{2}\left(t_{L, R}+k_{2 L, R}^{2}+k_{3 L, R}^{2}\right)=\frac{1}{2} t_{L, R}-\frac{1}{2}\left(M_{2 L, R}^{2}+M_{3 L, R}^{2}\right), \\
-k_{1 L, R} \cdot k_{3 L, R} & =-\sqrt{p^{2}+M_{1}^{2}} \cdot \sqrt{q^{2}+M_{3}^{2}}-p q \cos \phi-\vec{K}_{1 L, R} \cdot \vec{K}_{3 L, R} \\
& =\frac{1}{2}\left(u_{L, R}+k_{1 L, R}^{2}+k_{3 L, R}^{2}\right)=\frac{1}{2} u_{L, R}-\frac{1}{2}\left(M_{1 L, R}^{2}+M_{3 L, R}^{2}\right)
\end{aligned}
$$

where the left and the right Mandelstam variables are defined to be

$$
\begin{gathered}
s_{L, R} \equiv-\left(k_{1 L, R}+k_{2 L, R}\right)^{2}, \\
t_{L, R} \equiv-\left(k_{2 L, R}+k_{3 L, R}\right)^{2}, \\
u_{L, R} \equiv-\left(k_{1 L, R}+k_{3 L, R}\right)^{2},
\end{gathered}
$$

with

$$
s_{L, R}+t_{L, R}+u_{L, R}=\sum_{i} M_{i L, R}^{2}
$$

\section{B. Four-tachyon scatterings with $N_{R}=N_{L}=0$}

We are now ready to calculate the string scattering amplitudes. Let's first calculate the case with $N_{R}+N_{L}=0\left(\right.$ or $\left.N_{R}=N_{L}=0\right)$,

$$
\begin{aligned}
& A_{\text {closed }}^{\left(N_{R}+N_{L}=0\right)}(s, t, u) \\
& =\int d^{2} z \exp \left\{k_{1 L} \cdot k_{2 L} \ln z+k_{1 R} \cdot k_{2 R} \ln \bar{z}+k_{2 L} \cdot k_{3 L} \ln (1-z)+k_{2 R} \cdot k_{3 R} \ln (1-\bar{z})\right\} \\
& =\int d^{2} z \exp \left\{2 k_{1 R} \cdot k_{2 R} \ln |z|+2 k_{2 R} \cdot k_{3 R} \ln |1-z|\right. \\
& \left.\quad+\left(k_{1 L} \cdot k_{2 L}-k_{1 R} \cdot k_{2 R}\right) \ln z+\left(k_{2 L} \cdot k_{3 L}-k_{2 R} \cdot k_{3 R}\right) \ln (1-z)\right\} \\
& \quad
\end{aligned}
$$

where we have used $\alpha^{\prime}=2$ for closed string propagators

$$
\begin{aligned}
\left\langle X(z) X\left(z^{\prime}\right)\right\rangle & =-\frac{\alpha^{\prime}}{2} \ln \left(z-z^{\prime}\right), \\
\left\langle\tilde{X}(\bar{z}) \tilde{X}\left(\bar{z}^{\prime}\right)\right\rangle & =-\frac{\alpha^{\prime}}{2} \ln \left(\bar{z}-\bar{z}^{\prime}\right) .
\end{aligned}
$$


Note that for this simple case, Eq.(2.7) implies either $m=0$ or $n=0$. However, we will keep track of the general values of $(m, n)$ here for the reference of future calculations. By using the formula [25]

$$
\begin{aligned}
I & =\int \frac{d^{2} z}{\pi}|z|^{\alpha}|1-z|^{\beta} z^{n}(1-z)^{m} \\
& =\frac{\Gamma\left(-1-\frac{1}{2} \alpha-\frac{1}{2} \beta\right) \Gamma\left(1+n+\frac{1}{2} \alpha\right) \Gamma\left(1+m+\frac{1}{2} \beta\right)}{\Gamma\left(-\frac{1}{2} \alpha\right) \Gamma\left(-\frac{1}{2} \beta\right) \Gamma\left(2+n+m+\frac{1}{2} \alpha+\frac{1}{2} \beta\right)},
\end{aligned}
$$

we obtain

$$
\begin{aligned}
A_{\text {closed }}^{\left(N_{R}+N_{L}=0\right)}(s, t, u) \\
=\frac{\Gamma\left(-1-k_{1 R} \cdot k_{2 R}-k_{2 R} \cdot k_{3 R}\right) \Gamma\left(1+k_{1 L} \cdot k_{2 L}\right) \Gamma\left(1+k_{2 L} \cdot k_{3 L}\right)}{\Gamma\left(-k_{1 R} \cdot k_{2 R}\right) \Gamma\left(-k_{2 R} \cdot k_{3 R}\right) \Gamma\left(2+k_{1 L} \cdot k_{2 L}+k_{2 L} \cdot k_{3 L}\right)} \\
=\frac{\sin \left(-\pi k_{1 R} \cdot k_{2 R}\right) \sin \left(-\pi k_{2 R} \cdot k_{3 R}\right)}{\sin \left(-\pi-\pi k_{1 R} \cdot k_{2 R}-\pi k_{2 R} \cdot k_{3 R}\right)} \\
\\
\quad \cdot \frac{\Gamma\left(1+k_{1 R} \cdot k_{2 R}\right) \Gamma\left(1+k_{2 R} \cdot k_{3 R}\right)}{\Gamma\left(2+k_{1 R} \cdot k_{2 R}+k_{2 R} \cdot k_{3 R}\right)} \frac{\Gamma\left(1+k_{1 L} \cdot k_{2 L}\right) \Gamma\left(1+k_{2 L} \cdot k_{3 L}\right)}{\Gamma\left(2+k_{1 L} \cdot k_{2 L}+k_{2 L} \cdot k_{3 L}\right)} \\
\simeq \frac{\sin \left(\pi s_{L} / 2\right) \sin \left(\pi t_{R} / 2\right)}{\sin \left(\pi u_{L} / 2\right)} \frac{\Gamma\left(-1-\frac{t_{R}}{2}\right) \Gamma\left(-1-\frac{u_{R}}{2}\right)}{\Gamma\left(2+\frac{s_{R}}{2}\right)} \frac{\Gamma\left(-1-\frac{t_{L}}{2}\right) \Gamma\left(-1-\frac{u_{L}}{2}\right)}{\Gamma\left(2+\frac{s_{L}}{2}\right)},
\end{aligned}
$$

where we have used $M_{i L, R}^{2}=-2$ for $i=1,2,3,4$. In the above calculation, we have used the following well known formula for gamma function

$$
\Gamma(x)=\frac{\pi}{\sin (\pi x) \Gamma(1-x)} .
$$

\section{HIGH ENERGY BEHAVIORS}

\section{A. High energy massive scatterings for general $N_{R}+N_{L}$}

We now proceed to calculate the high energy scattering amplitudes for general higher mass levels with fixed $N_{R}+N_{L}$. With one compactified coordinate, the mass spectrum of the second vertex of the amplitude is

$$
M_{2}^{2}=\left(\frac{m_{2}^{2}}{R^{2}}+\frac{1}{4} n_{2}^{2} R^{2}\right)+N_{R}+N_{L}-2 .
$$

We now have more mass parameters to define the "high energy limit". So let's first clear

and redefine the concept of "high energy limit" in our following calculations. We are going to use three quantities $E^{2}, M_{2}^{2}$ and $N_{R}+N_{L}$ to define different regimes of "high energy 


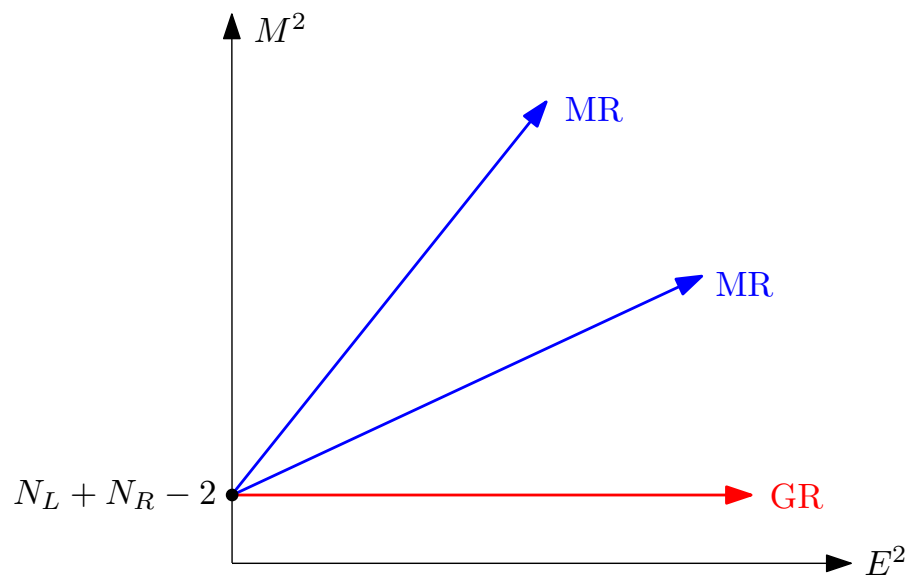

FIG. 1: Different regimes of "high energy limit". The high energy regime defined by $E^{2} \simeq M_{2}^{2} \gg$ $N_{R}+N_{L}$ will be called Mende regime (MR). The high energy regime defined by $E^{2} \gg M_{2}^{2}, E^{2} \gg$ $N_{R}+N_{L}$ will be called Gross regime (GR).

limit". See FIG. 1. The high energy regime defined by $E^{2} \simeq M_{2}^{2} \gg N_{R}+N_{L}$ will be called Mende regime (MR). The high energy regime defined by $E^{2} \gg M_{2}^{2}, E^{2} \gg N_{R}+N_{L}$ will be called Gross region (GR). In the high energy limit, the polarizations on the scattering plane for the second vertex operator are defined to be

$$
\begin{aligned}
e^{\mathbf{P}} & =\frac{1}{M_{2}}\left(\sqrt{p^{2}+M_{2}^{2}}, p, 0,0\right), \\
e^{\mathbf{L}} & =\frac{1}{M_{2}}\left(p, \sqrt{p^{2}+M_{2}^{2}}, 0,0\right), \\
e^{\mathbf{T}} & =(0,0,1,0)
\end{aligned}
$$

where the fourth component refers to the compactified direction. One can calculate the following formulas in the high energy limit

$$
\begin{aligned}
e^{\mathbf{P}} \cdot k_{1 L} & =e^{\mathbf{P}} \cdot k_{1 R}=-\frac{1}{M_{2}}\left(\sqrt{p^{2}+M_{1}^{2}} \sqrt{p^{2}+M_{2}^{2}}+p^{2}\right) \\
& =-\frac{p^{2}}{M_{2}}\left(2+\frac{M_{1}^{2}}{2 p^{2}}+\frac{M_{2}^{2}}{2 p^{2}}\right)+\mathcal{O}\left(p^{-2}\right), \\
e^{\mathbf{P}} \cdot k_{3 L} & =e^{\mathbf{P}} \cdot k_{3 R}=\frac{1}{M_{2}}\left(\sqrt{q^{2}+M_{3}^{2}} \sqrt{p^{2}+M_{2}^{2}}-p q \cos \phi\right) \\
& =\frac{p q}{M_{2}}\left[1-\cos \phi+\frac{M_{2}^{2}}{2 p^{2}}+\frac{M_{3}^{2}}{2 q^{2}}\right]+\mathcal{O}\left(p^{-2}\right),
\end{aligned}
$$




$$
\begin{aligned}
& e^{\mathbf{L}} \cdot k_{1 L}= e^{\mathbf{L}} \cdot k_{1 R}=-\frac{p}{M_{2}}\left(\sqrt{p^{2}+M_{1}^{2}}+\sqrt{p^{2}+M_{2}^{2}}\right) \\
&=-\frac{p^{2}}{M_{2}}\left(2+\frac{M_{1}^{2}}{2 p^{2}}+\frac{M_{2}^{2}}{2 p^{2}}\right)+\mathcal{O}\left(p^{-2}\right), \\
& e^{\mathbf{L}} \cdot k_{3 L}= e^{\mathbf{L}} \cdot k_{3 R}=\frac{1}{M_{2}}\left(p \sqrt{q^{2}+M_{3}^{2}}-q \sqrt{p^{2}+M_{2}^{2}} \cos \phi\right) \\
&= \frac{p q}{M_{2}}\left[1+\frac{M_{3}^{2}}{2 q^{2}}-\left(1+\frac{M_{2}^{2}}{2 p^{2}}\right) \cos \phi\right]+\mathcal{O}\left(p^{-2}\right), \\
& e^{\mathbf{T}} \cdot k_{1 L}=e^{\mathbf{T}} \cdot k_{1 R}=0, \\
& e^{\mathbf{T}} \cdot k_{3 L}=e^{\mathbf{T}} \cdot k_{3 R}=-q \sin \phi,
\end{aligned}
$$

which will be useful in the calculations of high energy string scattering amplitudes.

For the noncompactified open string, it was shown that [7, 8], at each fixed mass level $M_{o p}^{2}=2(N-1)$, a four-point function is at the leading order in high energy (GR) only for states of the following form

$$
|N, 2 l, q\rangle \equiv\left(\alpha_{-1}^{\mathbf{T}}\right)^{N-2 l-2 q}\left(\alpha_{-1}^{\mathbf{L}}\right)^{2 l}\left(\alpha_{-2}^{\mathbf{L}}\right)^{q}|0, k\rangle
$$

where $N \geqslant 2 l+2 q, l, q \geqslant 0$. To avoid the complicated subleading order calculation due to the $\alpha_{-1}^{\mathbf{L}}$ operator, we will choose the simple case $l=0$. We made a similar choice when dealing with the high energy string/D-brane scatterings [16, 17, 23]. There is still one complication in the case of compactified string due to the possible choices of $\alpha_{-n}^{25}$ and $\tilde{\alpha}_{-m}^{25}$ in the vertex operator. However, it can be easily shown that for each fixed mass level with given quantized and winding momenta $\left(\frac{m}{R}, \frac{1}{2} n R\right)$, and thus fixed $N_{R}+N_{L}$ level, vertex operators containing $\alpha_{-n}^{25}$ or $\tilde{\alpha}_{-m}^{25}$ are subleading order in energy in the high energy expansion compared to other choices $\alpha_{-1}^{\mathbf{T}}\left(\tilde{\alpha}_{-1}^{\mathbf{T}}\right)$ and $\alpha_{-2}^{\mathbf{L}}\left(\tilde{\alpha}_{-2}^{\mathbf{L}}\right)$ on the noncompact directions. In conclusion, in the calculation of compactified closed string in the GR, we are going to consider tensor state of the form

$$
\left|N_{L, R}, q_{L, R}\right\rangle \equiv\left(\alpha_{-1}^{\mathbf{T}}\right)^{N_{L}-2 q_{L}}\left(\alpha_{-2}^{\mathbf{L}}\right)^{q_{L}} \otimes\left(\tilde{\alpha}_{-1}^{\mathbf{T}}\right)^{N_{R}-2 q_{R}}\left(\tilde{\alpha}_{-2}^{\mathbf{L}}\right)^{q_{R}}|0\rangle
$$

at general $N_{R}+N_{L}$ level scattered from three other tachyon states with $N_{R}+N_{L}=0$.

Note that, in the GR, one can identify $e^{\mathbf{P}}$ with $e^{\mathbf{L}}$ as usual [4, 5]. However, in the MR, one can not identify $e^{\mathbf{P}}$ with $e^{\mathbf{L}}$. This can be seen from Eq.(3.5) to Eq.(3.8). In the MR, instead of using the tensor vertex in Eq.(3.12), we will use

$$
\left|N_{L, R}, q_{L, R}\right\rangle \equiv\left(\alpha_{-1}^{\mathbf{T}}\right)^{N_{L}-2 q_{L}}\left(\alpha_{-2}^{\mathbf{P}}\right)^{q_{L}} \otimes\left(\tilde{\alpha}_{-1}^{\mathbf{T}}\right)^{N_{R}-2 q_{R}}\left(\tilde{\alpha}_{-2}^{\mathbf{P}}\right)^{q_{R}}|0\rangle
$$


as the second vertex operator in the calculation of high energy scattering amplitudes. Note also that, in the MR, states in Eq.(3.13) may not be the only states which contribute to the high energy scattering amplitudes as in the GR. However, we will just choose these states to calculate the scattering amplitudes in order to compare with the corresponding high energy scattering amplitudes in the GR.

The high energy scattering amplitudes in the MR can be calculated to be

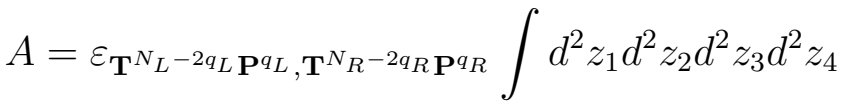

$$
\begin{aligned}
& \cdot\left\langle V_{1}\left(z_{1}, \bar{z}_{1}\right) V_{2}^{\mathbf{T}^{N_{L}-2 q_{L}} \mathbf{P}_{L}, \mathbf{T}^{N_{R}-2 q_{R}} \mathbf{P}^{q_{R}}}\left(z_{2}, \bar{z}_{2}\right) V_{3}\left(z_{3}, \bar{z}_{3}\right) V_{4}\left(z_{4}, \bar{z}_{4}\right)\right\rangle
\end{aligned}
$$

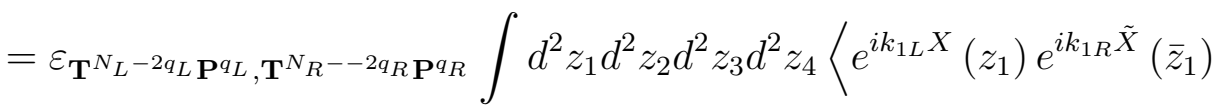

$$
\begin{aligned}
& \cdot\left(\partial X^{\mathbf{T}}\right)^{N_{L}-2 q_{L}}\left(i \partial^{2} X^{\mathbf{P}}\right)^{q_{L}} e^{i k_{2 L} X}\left(z_{2}\right)\left(\bar{\partial} \tilde{X}^{\mathbf{T}}\right)^{N_{R}-2 q_{R}}\left(i \bar{\partial}^{2} \tilde{X}^{\mathbf{P}}\right)^{q_{R}} e^{i k_{2 R} \tilde{X}}\left(\bar{z}_{2}\right) \\
& \left.e^{i k_{3 L} X}\left(z_{3}\right) e^{i k_{3 R} \tilde{X}}\left(\bar{z}_{3}\right) e^{i k_{4 L} X}\left(z_{4}\right) e^{i k_{4 R} \tilde{X}}\left(\bar{z}_{4}\right)\right\rangle \\
& =\int d^{2} z_{1} d^{2} z_{2} d^{2} z_{3} d^{2} z_{4} \cdot\left[\prod_{i<j}\left(z_{i}-z_{j}\right)^{k_{i L} \cdot k_{j L}}\left(\bar{z}_{i}-\bar{z}_{j}\right)^{k_{i R} \cdot k_{j R}}\right] \\
& \cdot\left[\frac{i e^{\mathbf{T}} \cdot k_{1 L}}{z_{1}-z_{2}}+\frac{i e^{\mathbf{T}} \cdot k_{3 L}}{z_{3}-z_{2}}+\frac{i e^{\mathbf{T}} \cdot k_{4 L}}{z_{4}-z_{2}}\right]^{N_{L}-2 q_{L}} \cdot\left[\frac{e^{\mathbf{P}} \cdot k_{1 L}}{\left(z_{1}-z_{2}\right)^{2}}+\frac{e^{\mathbf{P}} \cdot k_{3 L}}{\left(z_{3}-z_{2}\right)^{2}}+\frac{e^{\mathbf{P}} \cdot k_{4 L}}{\left(z_{4}-z_{2}\right)^{2}}\right]^{q_{L}} \\
& \cdot\left[\frac{i e^{\mathbf{T}} \cdot k_{1 R}}{\bar{z}_{1}-\bar{z}_{2}}+\frac{i e^{\mathbf{T}} \cdot k_{3 R}}{\bar{z}_{3}-\bar{z}_{2}}+\frac{i e^{\mathbf{T}} \cdot k_{4 R}}{\bar{z}_{4}-\bar{z}_{2}}\right]^{N_{R}-2 q_{R}} \cdot\left[\frac{e^{\mathbf{P}} \cdot k_{1 R}}{\left(\bar{z}_{1}-\bar{z}_{2}\right)^{2}}+\frac{e^{\mathbf{P}} \cdot k_{3 R}}{\left(\bar{z}_{3}-\bar{z}_{2}\right)^{2}}+\frac{e^{\mathbf{P}} \cdot k_{4 R}}{\left(\bar{z}_{4}-\bar{z}_{2}\right)^{2}}\right]^{q_{R}} .
\end{aligned}
$$

After the standard $S L(2, C)$ gauge fixing, we get

$$
\begin{aligned}
& A \simeq(-1)^{k_{1 L} \cdot k_{2 L}+k_{1 R} \cdot k_{2 R}+k_{1 L} \cdot k_{3 L}+k_{1 R} \cdot k_{3 R}+k_{2 L} \cdot k_{3 L}+k_{2 R} \cdot k_{3 R}} \\
& \cdot \int d^{2} z \cdot z^{k_{1 L} \cdot k_{2 L}} \cdot \bar{z}^{k_{1 R} \cdot k_{2 R}} \cdot(1-z)^{k_{2 L} \cdot k_{3 L}}(1-\bar{z})^{k_{2 R} \cdot k_{3 R}} \\
& \cdot\left[\frac{i e^{\mathbf{T}} \cdot k_{1 L}}{z}-\frac{i e^{\mathbf{T}} \cdot k_{3 L}}{1-z}\right]^{N_{L}-2 q_{L}} \cdot\left[\frac{i e^{\mathbf{T}} \cdot k_{1 R}}{\bar{z}}-\frac{i e^{\mathbf{T}} \cdot k_{3 R}}{1-\bar{z}}\right]^{N_{R}-2 q_{R}} \\
& \cdot\left[\frac{e^{\mathbf{P}} \cdot k_{1 L}}{z^{2}}+\frac{e^{\mathbf{P}} \cdot k_{3 L}}{(1-z)^{2}}\right]^{q_{L}} \cdot\left[\frac{e^{\mathbf{P}} \cdot k_{1 R}}{\bar{z}^{2}}+\frac{e^{\mathbf{P}} \cdot k_{3 R}}{(1-\bar{z})^{2}}\right]^{q_{R}} \text {. }
\end{aligned}
$$

By using Eqs.(3.5) to (3.10), the amplitude can be written as 


$$
\begin{aligned}
& A \sim(-1)^{n+q+q^{\prime}+k_{1 L} \cdot k_{2 L}+k_{1 R} \cdot k_{2 R}+k_{1 L} \cdot k_{3 L}+k_{1 R} \cdot k_{3 R}+k_{2 L} \cdot k_{3 L}+k_{2 R} \cdot k_{3 R}}(q \sin \phi)^{N_{L}+N_{R}-2 q_{L}-2 q_{R}} \\
& \int d^{2} z \cdot z^{k_{1 L} \cdot k_{2 L}} \cdot \bar{z}^{k_{1 R} \cdot k_{2 R}} \cdot(1-z)^{k_{2 L} \cdot k_{3 L}}(1-\bar{z})^{k_{2 R} \cdot k_{3 R}} \cdot\left[\frac{1}{1-z}\right]^{N_{L}-2 q_{L}}\left[\frac{1}{1-\bar{z}}\right]^{N_{R}-2 q_{R}} \\
& \cdot\left[\frac{-\frac{1}{M_{2}}\left(\sqrt{p^{2}+M_{1}^{2}} \sqrt{p^{2}+M_{2}^{2}}+p^{2}\right)}{z^{2}}+\frac{\frac{1}{M_{2}}\left(\sqrt{q^{2}+M_{3}^{2}} \sqrt{p^{2}+M_{2}^{2}}-p q \cos \phi\right)}{(1-z)^{2}}\right]^{q_{L}} \\
& \cdot\left[\frac{-\frac{1}{M_{2}}\left(\sqrt{p^{2}+M_{1}^{2}} \sqrt{p^{2}+M_{2}^{2}}+p^{2}\right)}{\bar{z}^{2}}+\frac{\frac{1}{M_{2}}\left(\sqrt{q^{2}+M_{3}^{2}} \sqrt{p^{2}+M_{2}^{2}}-p q \cos \phi\right)}{(1-\bar{z})^{2}}\right]^{q_{R}} \text {. } \\
& =(-1)^{k_{1 L} \cdot k_{2 L}+k_{1 R} \cdot k_{2 R}+k_{1 L} \cdot k_{3 L}+k_{1 R} \cdot k_{3 R}+k_{2 L} \cdot k_{3 L}+k_{2 R} \cdot k_{3 R}}(q \sin \phi)^{N_{L}+N_{R}}\left(\frac{1}{M_{2} q^{2} \sin ^{2} \phi}\right)^{q_{L}+q_{R}} \\
& \int d^{2} z \cdot z^{k_{1 L} \cdot k_{2 L}} \cdot \bar{z}^{k_{1 R} \cdot k_{2 R}} \cdot(1-z)^{k_{2 L} \cdot k_{3 L}}(1-\bar{z})^{k_{2 R} \cdot k_{3 R}} \cdot\left[\frac{1}{1-z}\right]^{N_{L}-2 q_{L}}\left[\frac{1}{1-\bar{z}}\right]^{N_{R}-2 q_{R}} \\
& \cdot \sum_{i=0}^{q} \sum_{j=0}^{q^{\prime}}\left(\begin{array}{l}
q \\
i
\end{array}\right)\left(\begin{array}{l}
q^{\prime} \\
j
\end{array}\right)\left(\frac{\sqrt{p^{2}+M_{1}^{2}} \sqrt{p^{2}+M_{2}^{2}}+p^{2}}{z^{2}}\right)^{i}\left(\frac{\sqrt{p^{2}+M_{1}^{2}} \sqrt{p^{2}+M_{2}^{2}}+p^{2}}{\bar{z}^{2}}\right)^{j} \\
& =(-1)^{k_{1 L} \cdot k_{2 L}+k_{1 R} \cdot k_{2 R}+k_{1 L} \cdot k_{3 L}+k_{1 R} \cdot k_{3 R}+k_{2 L} \cdot k_{3 L}+k_{2 R} \cdot k_{3 R}}(q \sin \phi)^{N_{L}+N_{R}} \\
& \cdot\left(-\frac{\sqrt{q^{2}+M_{3}^{2}} \sqrt{p^{2}+M_{2}^{2}}-p q \cos \phi}{M_{2} q^{2} \sin ^{2} \phi}\right)^{q_{L}+q_{R}} \\
& \cdot \sum_{i=0}^{q_{L}} \sum_{j=0}^{q_{R}}\left(\begin{array}{c}
q_{L} \\
i
\end{array}\right)\left(\begin{array}{c}
q_{R} \\
j
\end{array}\right)\left(\frac{\sqrt{p^{2}+M_{1}^{2}} \sqrt{p^{2}+M_{2}^{2}}+p^{2}}{-\sqrt{q^{2}+M_{3}^{2}} \sqrt{p^{2}+M_{2}^{2}}+p q \cos \phi}\right)^{i+j} \\
& \frac{\sin \left[-\pi\left(k_{1 R} \cdot k_{2 R}-2 j\right)\right] \sin \left[-\pi\left(k_{2 R} \cdot k_{3 R}-N_{R}+2 j\right)\right]}{\sin \left[-\pi\left(1+k_{1 R} \cdot k_{2 R}+k_{2 R} \cdot k_{3 R}-N_{R}\right)\right]} \\
& \cdot \frac{\Gamma\left(1+k_{1 R} \cdot k_{2 R}-2 j\right) \Gamma\left(1+k_{2 R} \cdot k_{3 R}-N_{R}+2 j\right)}{\Gamma\left(2+k_{1 R} \cdot k_{2 R}+k_{2 R} \cdot k_{3 R}-N_{R}\right)} \\
& \cdot \frac{\Gamma\left(1+k_{1 L} \cdot k_{2 L}-2 i\right) \Gamma\left(1+k_{2 L} \cdot k_{3 L}+2 i-N_{L}\right)}{\Gamma\left(2+k_{1 L} \cdot k_{2 L}+k_{2 L} \cdot k_{3 L}-N_{L}\right)},
\end{aligned}
$$

where, as in the calculation of section II for the GR, we have used Eq.(2.32) to do the 
integration. It is easy to do the following approximations for the gamma functions

$$
\begin{aligned}
& A \simeq(-1)^{k_{1 L} \cdot k_{2 L}+k_{1 R} \cdot k_{2 R}+k_{1 L} \cdot k_{3 L}+k_{1 R} \cdot k_{3 R}+k_{2 L} \cdot k_{3 L}+k_{2 R} \cdot k_{3 R}}(q \sin \phi)^{N_{L}+N_{R}} \\
& \cdot\left(-\frac{\sqrt{q^{2}+M_{3}^{2}} \sqrt{p^{2}+M_{2}^{2}}-p q \cos \phi}{M_{2} q^{2} \sin ^{2} \phi}\right)^{q_{L}+q_{R}} \\
& \cdot \sum_{i=0}^{q_{L}} \sum_{j=0}^{q_{R}}\left(\begin{array}{c}
q_{L} \\
i
\end{array}\right)\left(\begin{array}{c}
q_{R} \\
j
\end{array}\right)\left(\frac{\sqrt{p^{2}+M_{1}^{2}} \sqrt{p^{2}+M_{2}^{2}}+p^{2}}{-\sqrt{q^{2}+M_{3}^{2}} \sqrt{p^{2}+M_{2}^{2}}+p q \cos \phi}\right)^{i+j} \\
& \cdot \frac{\sin \left[-\pi k_{1 R} \cdot k_{2 R}\right] \sin \left[-\pi k_{2 R} \cdot k_{3 R}\right]}{\sin \left[-\pi\left(1+k_{1 R} \cdot k_{2 R}+k_{2 R} \cdot k_{3 R}\right)\right]} \\
& \cdot \frac{\Gamma\left(1+k_{1 R} \cdot k_{2 R}\right) \Gamma\left(1+k_{2 R} \cdot k_{3 R}\right) \Gamma\left(1+k_{1 L} \cdot k_{2 L}\right) \Gamma\left(1+k_{2 L} \cdot k_{3 L}\right)}{\Gamma\left(2+k_{1 R} \cdot k_{2 R}+k_{2 R} \cdot k_{3 R}\right) \Gamma\left(2+k_{1 L} \cdot k_{2 L}+k_{2 L} \cdot k_{3 L}\right)} \\
& \cdot \frac{\left(k_{1 R} \cdot k_{2 R}\right)^{-2 j}\left(k_{2 R} \cdot k_{3 R}\right)^{-N_{R}+2 j}}{\left(k_{1 L} \cdot k_{2 L}\right)^{-2 i}\left(k_{2 L} \cdot k_{3 L}\right)^{-N_{L}+2 i}} \\
&\left(k_{1 R} \cdot k_{2 R}+k_{2 R} \cdot k_{3 R}\right)^{-N_{R}} \frac{\left(k_{1 L} \cdot k_{2 L}+k_{2 L} \cdot k_{3 L}\right)^{-N_{L}}}{\left(k_{2}\right.} .
\end{aligned}
$$

One can now do the double summation and drop out the $M_{i L, R}$ terms to get

$$
\begin{aligned}
A \simeq & \left(-\frac{q \sin \phi\left(s_{L}+t_{L}\right)}{t_{L}}\right)^{N_{L}}\left(-\frac{q \sin \phi\left(s_{R}+t_{R}\right)}{t_{R}}\right)^{N_{R}}\left(\frac{1}{2 M_{2} q^{2} \sin ^{2} \phi}\right)^{q_{L}+q_{R}} \\
& \left.\cdot\left(\left(t_{R}-2 \vec{K}_{2 R} \cdot \vec{K}_{3 R}\right)+\frac{t_{R}^{2}\left(s_{R}-2 \vec{K}_{1 R} \cdot \vec{K}_{2 R}\right)}{s_{R}^{2}}\right)^{q_{R}}\right)^{q_{L}} \\
& \cdot\left(\left(t_{L}-2 \vec{K}_{2 L} \cdot \vec{K}_{3 L}\right)+\frac{t_{L}^{2}\left(s_{L}-2 \vec{K}_{1 L} \cdot \vec{K}_{2 L}\right)}{s_{L}^{2}}\right)^{-} \\
& \cdot \frac{\sin \left(\pi s_{L} / 2\right) \sin \left(\pi t_{R} / 2\right)}{\sin \left(\pi u_{L} / 2\right)} B\left(-1-\frac{t_{R}}{2},-1-\frac{u_{R}}{2}\right) B\left(-1-\frac{t_{L}}{2},-1-\frac{u_{L}}{2}\right) .
\end{aligned}
$$

Eq.(3.18) is valid for $E^{2} \gg N_{R}+N_{L}, M_{2}^{2} \gg N_{R}+N_{L}$.

\section{B. The infinite linear relations in the GR}

For the special case of GR with $E^{2} \gg M_{2}^{2}$, one can identify $q$ with $p$, and the amplitude in Eq.(3.18) further reduces to

$$
\begin{aligned}
\lim _{E^{2} \gg M_{2}^{2}} A & =\left(\frac{2 p \cos ^{3} \frac{\phi}{2}}{\sin \frac{\phi}{2}}\right)^{N_{L}+N_{R}}\left(-\frac{1}{2 M_{2}}\right)^{q_{L}+q_{R}} \frac{\sin \left(\pi s_{L} / 2\right) \sin \left(\pi t_{R} / 2\right)}{\sin \left(\pi u_{L} / 2\right)} \\
& \cdot B\left(-1-\frac{t_{R}}{2},-1-\frac{u_{R}}{2}\right) B\left(-1-\frac{t_{L}}{2},-1-\frac{u_{L}}{2}\right) .
\end{aligned}
$$


It is crucial to note that the high energy limit of the beta function with $s+t+u=2 n-8$ is $[4]$

$$
\begin{aligned}
B\left(-1-\frac{t}{2},-1-\frac{u}{2}\right) & =\frac{\Gamma\left(-\frac{t}{2}-1\right) \Gamma\left(-\frac{u}{2}-1\right)}{\Gamma\left(\frac{s}{2}+2\right)} \\
& \simeq E^{-1-2 n}\left(\sin \frac{\phi}{2}\right)^{-3}\left(\cos \frac{\phi}{2}\right)^{5-2 n} \\
& \cdot \exp \left(-\frac{t \ln t+u \ln u-(t+u) \ln (t+u)}{2}\right)
\end{aligned}
$$

where we have calculated the approximation up to the next leading order in energy $E$. Note that the appearance of the prepower factors in front of the exponential fall-off factor. For our purpose here, with Eq.(2.28), we have

$$
s_{L, R}+t_{L, R}+u_{L, R}=\sum_{i} M_{i L, R}^{2}=2 N_{L, R}-8,
$$

and the high energy limit of the beta functions in Eq.(3.19) can be further calculated to be

$$
\begin{aligned}
& B\left(-1-\frac{t_{R}}{2},-1-\frac{u_{R}}{2}\right) B\left(-1-\frac{t_{L}}{2},-1-\frac{u_{L}}{2}\right) \\
& \simeq E^{-1-2\left(N_{L}+N_{R}\right)}\left(\sin \frac{\phi}{2}\right)^{-3}\left(\cos \frac{\phi}{2}\right)^{5-2\left(N_{L}+N_{R}\right)} \\
& \cdot \exp \left(-\frac{t_{L} \ln t_{L}+u_{L} \ln u_{L}-\left(t_{L}+u_{L}\right) \ln \left(t_{L}+u_{L}\right)}{2}\right) \\
& \cdot \exp \left(-\frac{t_{R} \ln t_{R}+u_{R} \ln u_{R}-\left(t_{R}+u_{R}\right) \ln \left(t_{R}+u_{R}\right)}{2}\right) \\
& \simeq E^{-1-2\left(N_{L}+N_{R}\right)}\left(\sin \frac{\phi}{2}\right)^{-3}\left(\cos \frac{\phi}{2}\right)^{5-2\left(N_{L}+N_{R}\right)} \exp \left(-\frac{t \ln t+u \ln u-(t+u) \ln (t+u)}{4}\right),
\end{aligned}
$$

where we have implicitly used the relation $\alpha_{\text {closed }}^{\prime}=4 \alpha_{\text {open }}^{\prime}=2$. By combining Eq.(3.19) and Eq.(3.22), we end up with

$$
\begin{aligned}
\lim _{E^{2} \gg M_{2}^{2}} A & \simeq\left(-\frac{2 \cot \frac{\phi}{2}}{E}\right)^{N_{L}+N_{R}}\left(-\frac{1}{2 M_{2}}\right)^{q_{L}+q_{R}} E^{-1}\left(\sin \frac{\phi}{2}\right)^{-3}\left(\cos \frac{\phi}{2}\right)^{5} \\
& \cdot \frac{\sin \left(\pi s_{L} / 2\right) \sin \left(\pi t_{R} / 2\right)}{\sin \left(\pi u_{L} / 2\right)} \exp \left(-\frac{t \ln t+u \ln u-(t+u) \ln (t+u)}{4}\right) .
\end{aligned}
$$

We see that there is a $\left(\frac{m}{R}, \frac{1}{2} n R\right)$ dependence in the $\frac{\sin \left(\pi s_{L} / 2\right) \sin \left(\pi t_{R} / 2\right)}{\sin \left(\pi u_{L} / 2\right)}$ factor in our final result. This is physically consistent as one expects a $\left(\frac{m}{R}, \frac{1}{2} n R\right)$ dependent Regge-pole and zero structures in the high energy string scattering amplitudes. In conclusion, in the GR, 
for each fixed mass level with given quantized and winding momenta $\left(\frac{m}{R}, \frac{1}{2} n R\right)$ (thus fixed $N_{L}$ and $N_{R}$ by Eq.(2.7)), we have obtained infinite linear relations among high energy scattering amplitudes of different string states with various $\left(q_{L}, q_{R}\right)$. Note also that this

result reproduces the correct ratios $\left(-\frac{1}{2 M_{2}}\right)^{q_{L}+q_{R}}$ obtained in the previous works [16, 17, 23]. However, the mass parameter $M_{2}$ here depends on $\left(\frac{m}{R}, \frac{1}{2} n R\right)$. It is also interesting to see that, if not for the $\left(\frac{m}{R}, \frac{1}{2} n R\right)$ dependence in the $\frac{\sin \left(\pi s_{L} / 2\right) \sin \left(\pi t_{R} / 2\right)}{\sin \left(\pi u_{L} / 2\right)}$ factor in the high energy scattering amplitudes in the GR, we would have had a linear relation among scattering amplitudes of different string states in different mass levels with fixed $\left(N_{R}+N_{L}\right)$.

Presumably, the infinite linear relations obtained above can be reproduced by using the method of high energy ZNS, or high energy Ward identities, adopted in the previous works [4, 5, 6, 7, 8, 9, 10, 11]. The existence of Soliton ZNS at arbitrary mass levels was constructed in [22]. A closer look in this direction seems worthwhile. In the paper, however, we are more interested in understanding the power-law behavior of the high energy string scattering amplitudes and breakdown of the infinite linear relations as we will discuss in the next section.

\section{Power-law and breakdown of the infinite linear relations in the MR}

In this section we discuss the power-law behavior of high energy string scattering amplitudes in a compact space. We will see that, in the MR, the infinite linear relations derived in section IIIB break down and, simultaneously, the UV exponential fall-off behavior of high energy string scattering amplitudes enhances to power-law behavior. The power-law behavior of high energy string scatterings in a compact space was first suggested by Mende [20]. Here we give a mathematically more concrete description. It is easy to see that the "power law" condition, i.e. Eq.(3.7) in Mende's paper [20],

$$
k_{1 L} \cdot k_{2 L}+k_{1 R} \cdot k_{2 R}=\text { constant }
$$


turns out to be

$$
\begin{aligned}
& -\left(k_{1 L} \cdot k_{2 L}+k_{1 R} \cdot k_{2 R}\right) \\
& =\sqrt{p^{2}+M_{1}^{2}} \cdot \sqrt{p^{2}+M_{2}^{2}}+p^{2}+\left(\vec{K}_{1 L} \cdot \vec{K}_{2 L}+\vec{K}_{1 R} \cdot \vec{K}_{2 R}\right) \\
& =\sqrt{p^{2}+M_{1}^{2}} \cdot \sqrt{p^{2}+M_{2}^{2}}+p^{2}+2\left(\vec{K}_{1} \cdot \vec{K}_{2}+\vec{L}_{1} \cdot \vec{L}_{2}\right) \\
& =\text { constant. }
\end{aligned}
$$

As $p \rightarrow \infty$, due to the existence of winding modes in the compactified closed string, it is possible to choose $\left(\vec{K}_{1}, \vec{K}_{2} ; \vec{L}_{1}, \vec{L}_{2}\right)$ such that

$$
\vec{K}_{1} \cdot \vec{K}_{2}+\vec{L}_{1} \cdot \vec{L}_{2}<0
$$

and let $\left(\vec{K}_{1} \cdot \vec{K}_{2}+\vec{L}_{1} \cdot \vec{L}_{2}\right) \rightarrow-\infty$ to make

$$
\begin{aligned}
k_{1 L} \cdot k_{2 L} & +k_{1 R} \cdot k_{2 R} \simeq \text { constant } \\
\Rightarrow s_{L}+s_{R} & \simeq \text { constant }
\end{aligned}
$$

In our calculation, this condition implies the beta functions in Eq.(3.18) reduce to

$$
\begin{aligned}
& B\left(-1-\frac{t_{R}}{2},-1-\frac{u_{R}}{2}\right) B\left(-1-\frac{t_{L}}{2},-1-\frac{u_{L}}{2}\right) \\
& =\frac{\Gamma\left(-\frac{t_{R}}{2}-1\right) \Gamma\left(-\frac{u_{R}}{2}-1\right) \Gamma\left(-\frac{t_{L}}{2}-1\right) \Gamma\left(-\frac{u_{L}}{2}-1\right)}{\Gamma\left(\frac{s_{R}}{2}+2\right)} \\
& =\frac{\sin \left(\pi s_{R} / 2\right) \Gamma\left(-\frac{t_{R}}{2}-1\right) \Gamma\left(-\frac{u_{R}}{2}-1\right) \Gamma\left(-\frac{t_{L}}{2}-1\right) \Gamma\left(-\frac{u_{L}}{2}-1\right)}{\pi \frac{s_{R}}{2}\left(1+\frac{s_{R}}{2}\right)\left(-1+\frac{s_{R}}{2}\right)},
\end{aligned}
$$

which behaves as power-law in the high energy limit! On the other hand, it is obvious that the $\left(q_{L}, q_{R}\right)$ dependent power factors of the amplitude in Eq.(3.18)

$$
\begin{aligned}
A_{q_{L}, q_{R}} \simeq & \left(\frac{1}{2 M_{2} q^{2} \sin ^{2} \phi}\right)^{q_{L}+q_{R}} \\
& \cdot\left(\left(t_{R}-2 \vec{K}_{2 R} \cdot \vec{K}_{3 R}\right)+\frac{t_{R}^{2}\left(s_{R}-2 \vec{K}_{1 R} \cdot \vec{K}_{2 R}\right)}{s_{R}^{2}}\right)^{q_{R}} \\
& \cdot\left(\left(t_{L}-2 \vec{K}_{2 L} \cdot \vec{K}_{3 L}\right)+\frac{t_{L}^{2}\left(s_{L}-2 \vec{K}_{1 L} \cdot \vec{K}_{2 L}\right)}{s_{L}^{2}}\right)^{q_{L}} .
\end{aligned}
$$

show no linear relations in the MR. This is very different from the case of high energy scattering amplitude in Eq.(3.23) in the GR, which shows nice linear relations

$$
A_{q_{L}, q_{R}} \simeq\left(-\frac{1}{2 M_{2}}\right)^{q_{L}+q_{R}} .
$$


Note that the mechanism to break the linear relations and the mechanism to enhance the amplitude to power-law are all due to $E \simeq M_{2}$ in the MR. In our notation, Eq.(3.24) is equivalent to the following condition

$$
\lim _{p \rightarrow \infty} \frac{\sqrt{p^{2}+M_{1}^{2}} \cdot \sqrt{p^{2}+M_{2}^{2}}+p^{2}}{\vec{K}_{1} \cdot \vec{K}_{2}+\vec{L}_{1} \cdot \vec{L}_{2}} \sim \frac{E^{2}}{\left(\frac{m_{1} m_{2}}{R^{2}}+\frac{1}{4} n_{1} n_{2} R^{2}\right)} \sim-\mathcal{O}(1) .
$$

For our purpose here, as we will see soon, it is good enough to choose only one compactified coordinate to realize Eq.(3.32). First of all, in addition to Eq.(2.17) and Eq.(2.18), Eq.(2.7) implies

$$
m_{i} n_{i}=0, i=1,2,3,4(\text { no sum on } i) .
$$

This is because three of the four vertex are tachyons. Also, since we are going to take $n_{2}$ to infinity with fixed $N_{R}+N_{L}$ in order to satisfy Eq.(3.32), we are forced to take $m_{2}=0$. In sum, we can take, say, $m_{i}=0$ for $i=1,2,3,4$, and $n_{1}=-n_{2}=-n, n_{3}=-2 n, n_{4}=0$, and then let $n \rightarrow \infty$ to realize Eq.(3.32). Note that it is crucial to choose different sign for $n_{1}$ and $n_{2}$ in order to achieve the minus sign in Eq.(3.32). We stress that there are other choices to realize the condition. One notes that all choices implies

$$
N_{R}=N_{L}
$$

It is obvious that one can also compactify more than one coordinate to realize the Mende condition. We conclude that the high energy scatterings of the "highly winding string states" of the compactified closed string in the MR behave as the unusual UV power-law, and the usual linear relations among scattering amplitudes break down due to the unusual power-law behavior.

\section{CONCLUSION}

In this paper we calculate high energy scattering amplitudes of closed bosonic string compactified on torus. We define two regimes of high energy limit, the Gross regime (GR) and the Mende regime (MR). In the GR, for each fixed mass level with given quantized and winding momenta $\left(\frac{m}{R}, \frac{1}{2} n R\right)$, we obtain infinite linear relations among high energy scattering amplitudes of different string states. In the MR, we discover that linear relations with $N_{R}=N_{L}$ break down and, simultaneously, the amplitudes enhance to power-law behavior 
instead of the usual exponential fall-off behavior at high energies. The result of this work gives a concrete example to justify the coexistence of the linear relations and the softer exponential fall-off behavior of high energy string scattering amplitudes as we pointed out previously [17, 23]. It is also reminiscent of our previous work on the power-law behavior of high energy string/domain-wall scatterings [17]. However, in the case of string/domain-wall scatterings, the high energy scattering amplitudes behaves as power-law for the whole UV kinematic regime, and one can not see the transition from UV power-law behavior to the UV exponential fall-off behavior, neither can one see the transition from nonlinear relations to the linear relations among high energy scattering amplitudes of different string states. On the contrary, for the high energy scatterings of the compactified closed string considered in this paper, one gets more kinematic variables, namely $\left(\frac{m}{R}, \frac{1}{2} n R\right)$, to cover both GR and MR, so that one can see this interesting transition. Through the observation of this transition, one further confirms that the infinite linear relations obtained in [4, 5, 6, 7, 8, 9, 10, 11] are responsible for the UV softer string scattering amplitudes than the field theory scatterings.

\section{Acknowledgments}

We would like to thank the hospitality of University of Tokyo at Komaba, where this work is finalized. We are indebted to Chuan-Tsung Chan, Koji Hashimoto, Yoichi Kazama, Akitsugs Miwa, Yu Nakayama, Naoto Yokoi and Tamiaki Yoneya for many of their enlightening discussions. This work is supported in part by the National Science Council, 50 billions project of Ministry of Educaton and National Center for Theoretical Science, Taiwan, R.O.C.

[1] D. J. Gross and P. F. Mende, Phys. Lett. B 197, 129 (1987); Nucl. Phys. B 303, 407 (1988).

[2] D. J. Gross, Phys. Rev. Lett. 60, 1229 (1988); Phil. Trans. R. Soc. Lond. A329, 401 (1989).

[3] D. J. Gross and J. L. Manes, Nucl. Phys. B 326, 73 (1989). See section 6 for details.

[4] C. T. Chan and J. C. Lee, Phys. Lett. B 611, 193 (2005). J. C. Lee, arXiv:hep-th/0303012.

[5] C. T. Chan and J. C. Lee, Nucl. Phys. B 690, 3 (2004).

[6] C. T. Chan, P. M. Ho and J. C. Lee, Nucl. Phys. B 708, 99 (2005).

[7] C. T. Chan, P. M. Ho, J. C. Lee, S. Teraguchi and Y. Yang, Nucl. Phys. B 725, 352 (2005). 
[8] C. T. Chan, P. M. Ho, J. C. Lee, S. Teraguchi and Y. Yang, Phys. Rev. Lett. 96 (2006) 171601.

[9] C. T. Chan, P. M. Ho, J. C. Lee, S. Teraguchi and Y. Yang, Nucl. Phys. B 749, 266 (2006).

[10] C. T. Chan, J. C. Lee and Y. Yang, Nucl. Phys. B 738, 93 (2006).

[11] C. T. Chan, J. C. Lee and Y. Yang, Nucl. Phys. B 749, 280 (2006).

[12] Pei-Ming Ho, Xue-Yan Lin, Phys.Rev. D73 (2006) 126007.

[13] J. C. Lee, Phys. Lett. B 241, 336 (1990); Phys. Rev. Lett. 64, 1636 (1990). J. C. Lee and B. Ovrut, Nucl. Phys. B 336, 222 (1990); J.C.Lee, Phys. Lett. B 326, 79 (1994).

[14] T. D. Chung and J. C. Lee, Phys. Lett. B 350, 22 (1995). Z. Phys. C 75, 555 (1997). J. C. Lee, Eur. Phys. J. C 1, 739 (1998).

[15] H. C. Kao and J. C. Lee, Phys. Rev. D 67, 086003 (2003). . C. T. Chan, J. C. Lee and Y. Yang, Phys. Rev. D 71, 086005 (2005)

[16] C. T. Chan, J. C. Lee and Y. Yang, " Scatterings of massive string states from D-brane and their linear relations at high energies", Nucl.Phys.B764, 1 (2007).

[17] C. T. Chan, J. C. Lee and Y. Yang, "Power-law Behavior of Strings Scattered from Domainwall and Breakdown of Their High Energy Linear Relations", hep-th/0610219.

[18] For a review, see A. Hashimoto and I.R. Klebanov, "Scattering of strings from D-branes" hep-th/9611214.

[19] M.R. Garousi and R.C. Myers, "Superstring scattering from D-Branes" Nucl. Phys. B475 (1996) 193, hep-th/9603194.

[20] Paul F. Mende, "High Energy String Collisions in a Compact Space", Phys.Lett. B326 (1994) 216, hep-th/9401126

[21] Ramzi R. Khuri, "Veneziano Amplitude for Winding Strings", Phys.Rev. D48 (1993) 2823, hep-th/9303074.

[22] Jen-Chi Lee, "Soliton gauge states and T-duality of closed Bosonic string compactified on torus", Eur.Phys.C7,669 (1999)

[23] J.C. Lee and Y. Yang, "Linear Relations of High Energy Absorption/Emission Amplitudes of D-brane", Phys.Lett. B646 (2007) 120, hep-th/0612059.

[24] M. B. Green, J. H. Schwarz and E. Witten, "Superstring Theory. Vol. 1," Cambridge University Press 1987.

[25] D. Gross, J. Harvey, E. Martinec, and R. Rohm, "Heterotic string theory II", Nucl. Phys. B267 (1986) 75. 